\title{
Nickel Thin Film Resonantly Generated at a Rate of Megahertz
}

\author{
M. Saitou \\ Department of Mechanical Systems Engineering, University of the Ryukyus, 1 Senbaru Nishihara-cho \\ Okinawa, 903-0213, Japan \\ E-mail: saitou@tec.u-ryukyu.ac.jp
}

doi: $10.20964 / 2016.07 .34$

Received: 4 April 2016 / Accepted: 13 May 2016 / Published: 4 June 2016

\begin{abstract}
Resonant frequencies at which masses of Ni thin films deposited from a nickel sulfamate solution significantly increase are observed using a rectangular pulse current technique having a frequency of the megahertz scale. The resonant frequency spacing between the neighboring resonant frequencies keeps a constant value of $0.2 \mathrm{MHz}$. The presence of many resonant frequencies and the constant resonant frequency spacing show an energy level transition between an electron at the Fermi energy level in an electrode and a nickel sulfamate ion characterized by a quantized rotational energy level. In addition, at a frequency beyond the highest resonant frequency the normalized deposition mass that keeps a constant value nearly equal to 1 is found. The frequency named as a non-capacitative frequency shifts to a lower frequency with an increase in the solution temperature. This indicates that the electric double layer in the solution loses a capacitative property owing to nickel sulfamate ions that do not rotate at the applied pulse frequency.
\end{abstract}

Keywords: resonant frequency; electrodeposition; energy level transition; Ni thin film; quantized rotational energy level

\section{$\underline{\text { FULL TEXT }}$}

(C) 2016 The Authors. Published by ESG (www.electrochemsci.org). This article is an open access article distributed under the terms and conditions of the Creative Commons Attribution license (http://creativecommons.org/licenses/by/4.0/). 